\title{
Optimus: Towards Optimal Layer-Fusion on Deep Learning Processors
}

\author{
Xuyi Cai \\ Institute of Computing Technology, \\ Chinese Academy of Sciences \\ University of Chinese Academy of \\ Sciences \\ Beijing, China \\ caixuyi20b@ict.ac.cn
}

\author{
Ying Wang* \\ State Key Laboratory of Computer \\ Architecture \\ Institute of Computing Technology, \\ Chinese Academy of Sciences \\ Beijing, China \\ wangying2009@ict.ac.cn
}

\author{
Lei Zhang ${ }^{\dagger}$ \\ Institute of Computing Technology, \\ Chinese Academy of Sciences \\ Beijing, China \\ zlei@ict.ac.cn
}

\begin{abstract}
Neural network layer fusion has been proposed to parallelize the inference of neural layers and thus significantly reduces the feature-induced memory accesses. However, how to fuse the neural layers is still a challenging issue that heavily depends on both the network architecture and the specific DNN processor configuration. This work formalizes the layer fusion problem for DNN processors, proves that prior fusion solutions cannot guarantee memory-level optimality, and presents a novel neural network fusion framework, Optimus. Optimus includes an accurate memory cost model to evaluate fusion schemes, and a Computing-Graph (CG) based layer fusion algorithm, which generates high-efficiency layer-fusion schemes for arbitrary network architectures on DNN processors. The proposed off-line and on-line graph-based fusion algorithms can reduce $10.1 \%-72.2 \%$ off-chip memory traffic and obtain $1.71 \mathrm{x}-3.94 \mathrm{x}$ energy efficiency over SOTA baselines on DNN workloads, and they bring significant power-efficiency boost to the DNN processors of different architectures and dataflows.
\end{abstract}

CCS Concepts: • Hardware $\rightarrow$ Emerging languages and compilers; Emerging tools and methodologies; • Computer systems organization $\rightarrow$ Neural networks; Embedded systems.

Keywords: neural network, embedded processor, memory, layer fusion

\footnotetext{
${ }^{*}$ Corresponding author.

${ }^{\dagger}$ Lei Zhang is also with Jeejio (Ningbo) Technology Co., Ltd.
}

Permission to make digital or hard copies of all or part of this work for personal or classroom use is granted without fee provided that copies are not made or distributed for profit or commercial advantage and that copies bear this notice and the full citation on the first page. Copyrights for components of this work owned by others than ACM must be honored. Abstracting with credit is permitted. To copy otherwise, or republish, to post on servers or to redistribute to lists, requires prior specific permission and/or a fee. Request permissions from permissions@acm.org.

LCTES '21, fune 22, 2021, Virtual, Canada

(C) 2021 Association for Computing Machinery.

ACM ISBN 978-1-4503-8472-8/21/06 ..\$15.00

https://doi.org/10.1145/3461648.3463848

\section{ACM Reference Format:}

Xuyi Cai, Ying Wang, and Lei Zhang. 2021. Optimus: Towards Optimal Layer-Fusion on Deep Learning Processors. In Proceedings of the 22nd ACM SIGPLAN/SIGBED International Conference on Languages, Compilers, and Tools for Embedded Systems (LCTES '21), Fune 22, 2021, Virtual, Canada. ACM, New York, NY, USA, 13 pages. https://doi.org/10.1145/3461648.3463848

\section{Introduction}

Deep neural networks (DNNs) have been applied widely in the machine learning field, such as computer vision, NLP, speech recognition, etc. Particularly, the confluence of deep learning technologies and IoT devices has propelled the development of novel intelligent applications. However, the computational and memory overhead induced by DNNs poses a significant challenge to the embedded usage. To address this issue, there are plenty of specialized deep learning processors or $\mathrm{CNN}$ accelerators developed to highly offer energy-efficient and less-expensive hardware solutions.

Early deep learning processors and systems put more emphasis on the compression of neural parameters to decrease the memory and computational overhead of network inference for embedded usage [6, 8-10]. However, with the development of deep learning algorithms, state-of-the-art lightweight network architectures [14-16, 40] exhibit a clear design trend that the size of intermediate feature maps (fmaps) generated by neural layers is growing at a much faster pace than the parameter size. In this context, the technology of layer fusion [1] is becoming more and more important for future deep learning processors. As illustrated in Fig. 1, the DNN model is represented as computational directed acyclic graphs (CDAGs), and the layer fusion technique divides the network model into fused layer-groups (G1, G2, G3, G4). Within a fused layer-group, the intermediate feature data could be consumed immediately after being produced rather than being evicted to the off-chip memory. Without layer fusion, the intermediate data must be re-loaded again from the main memory to the compact on-chip memory before the next layer is started. Thus, it is viable to employ the layer fusion technique to improve the performance of many existing deep learning processors and reduce the memory 


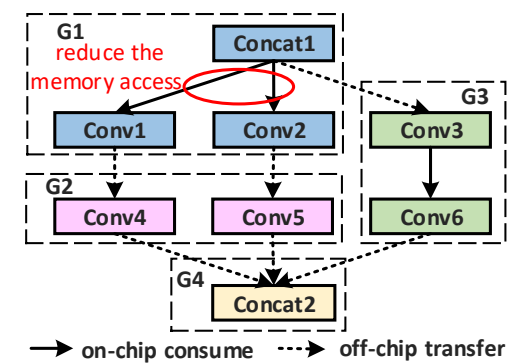

Figure 1. Example of CG-based layer fusion.

access overhead. Although there are some previous works $[1,29,30,37]$ focused on layer fusion for DNN processors, to achieve the optimal layer fusion remains an unaddressed and non-trivial problem.

First, previous layer fusion works do not allow parametersinduced memory accesses when processing the fused layergroups, i.e., all the depended parameters of a fused layergroup must be kept in the on-chip memory. However, it is very common for a given off-the-shelf DNN processor to fall short of on-chip parameter buffer space for the fused layergroups. Thus, it is quite important to precisely measure the reachable minimum memory access overhead, including both feature-map and parameters induced memory accesses, for a fused layer-group, when we seek the layer fusion strategy that aims to reach the memory optimality.

Second, the design space of layer fusion is massive, and it is an NP-hard problem [20] to explore the entire layer fusion space for a complicated NN model. Many previous works attempted to explore how to reach better layer fusion results in a restricted design space. However, to search for the optimal layer fusion result must includes and evaluate all the possible options of fused layer-groups.

Third, the other important aspect of prior works on network fusion is that how to reach fast and high-performance online layer fusion is not discussed, since most of them employ expensive search polices or enumeration methods to find premier fusion schemes offline. However, for the scenarios and platforms such as reconfigurable processor architecture [39], resource sharing, consolidation and FPGA virtualization for AI workloads [32], the underlying computing resources are dynamic, and a fixed fusion scheme does not work when the memory space or processing elements assigned to the network workload are changing, since the optimal solution depends on the resource assignment. In this case, searching for the optimal fusion must be performed online, and it must be fast enough and put negligible impacts on the end-to-end network inference latency.

In general, none of the previous works on neural network fusion has resolved the problem about how to reach the optimal layer fusion schemes for arbitrary network architectures on the given deep learning processors. In

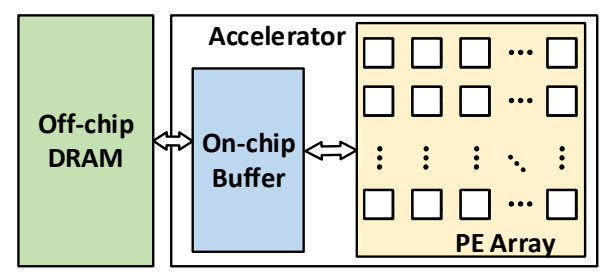

Figure 2. A typical DNN processor architecture design.

this work, we propose a layer fusion framework, Optimus ${ }^{1}$, to find the true memory-optimal layer fusion solution for an arbitrary combination of networks and DNN processors. The key contributions of this paper are:

- We for the first time present an accurate memorycost model for the evaluation of layer fusion schemes, which is useful in capturing the achievable minimum off-chip memory overhead for the fused layer-groups. Based on the proposed memory-cost function, we derive the optimal Computing-Graph (CG) based layer fusion algorithm and also prove that the discovered fusion solutions guarantee the minimum memory overhead for the networks running on the specific processors.

- We propose the optimal off-line hardware-aware layer fusion algorithm (HaFS) upon the computational graph of DNNs, based on dynamic programming (DP), which explores all the feasible layer fusion options to find the optimal solution.

- We introduce a fast on-line layer fusion algorithm (OnFS) to find the near-optimal fusion scheme for the online scenario when the resources of processors are allocated dynamically.

- The experiments are conducted across a variety of neural network architectures and network processors. The results show that the proposed algorithms guarantee the memory optimality and bring significant benefits to DNN processors of different architectures and dataflows when compared to the baselines.

\section{Background and Motivation}

\subsection{DNN Processor}

The rise of DNNs $[14,16,19]$ has stimulated intensive researches on deep learning processors [6, 8-10] which are developed to enable more energy-efficient hardware solutions than general-purpose CPUs and GPUs. Fig. 2 exemplifies a typical architecture of state-of-the-art DNN processors, which consists of a PE-array, an on-chip buffer, and a memory controller connected to the off-chip memory module (e.g., DRAM). The on-chip memory can be used to cache the reusable on-chip data to reduce off-chip memory accesses. However, most processors usually have such compact onchip memory for neural parameters and activations that the

\footnotetext{
${ }^{1}$ https://github.com/SheaCai/optimus
} 
data exchange between off-chip memory and on-chip buffer is frequent. Furthermore, the energy cost of DRAM access is orders of magnitude higher than that of the requests hitting other levels [8], thereby dominating the system energy consumption of typical neural network chips.

Moreover, the resources of some processors for the target workload are not always fixed, and they can be allocated ondemand for the multi-tenant use cases, which includes the scenarios of resource-sharing IaaS cloud and elastic systems [3, 39], e.g., heterogeneous SoCs with shared memory, reconfigurable processors and accelerators [39], FPGA virtualization and consolidation for DL [32]. Thus, fast and efficient online redeployment of the neural models is also necessary to ensure that the task progresses will not be affected even in the case of frequent dynamic resource reallocation [32].

\subsection{Neural Network Layer Fusion}

Layer fusion is to execute a group of adjacent neural network operators in parallel, so that the intermediate results generated by one operator can be consumed immediately without being evicted to the off-chip memory. Fig. 3 exemplifies the execution process of a fused layer-group with two convolution (Conv) layers, e.g., G3 in Fig. 1 . For the $l$-th Conv layer of a certain fused layer-group, the height/width of the output feature map (ofmap) is $O_{h}^{(l)} \times O_{w}^{(l)}$ and the size of the weight kernel is $K_{h}^{(l)} \times K_{w}^{(l)}$. Additionally, $C_{i n}, C_{\text {out }}$ are the channel numbers of input and output feature map respectively. The convolution strides in the $h$ and $w$ directions are $S_{h}^{(l)}$ and $S_{w}^{(l)}$, respectively. Besides the Conv layer, other layers can also be expressed by these factors. For example, a fully-connected (FC) layer can be considered as a special Conv layer by setting $S_{h}^{(l)}, S_{w}^{(l)}, O_{w}^{(l)}, O_{h}^{(l)}, K_{w}^{(l)}$ and $K_{h}^{(l)}$ to 1 .

As illustrated in Fig. 3, a fmap tile $t_{h}^{(l)} \times t_{w}^{(l)}$ is a partition from one fmap and it is the basic unit received and processed by the DNN processors. In the fused layer-group, the fmap tiles are directly passed to the subsequent layers without being evicted to the off-chip memory. The height/width of the ofmap tiles between two layers satisfy the equation $t^{(l-1)}=t^{(l)} \cdot S^{(l)}+K^{(l)}-S^{(l)}[1]$, wherein layer- $(l-1)$ is the input producer of layer- $l$. Thus, according to the producerand-consumer relationship, the required minimum size of the ofmap tile for layer- $l$ in the fused-group, which is referred to as $\mathcal{R}\left(l, t^{(n)}\right)$, can be deduced and determined backwardly from the last layer (layer- $n$ ) in the group. For example, as shown in in Fig. 3, to output one single pixel in layer-2 depends on $3 \times 3 \times 2$ output pixels from layer-1, which further relies on $5 \times 5 \times 2$ output pixels from layer-0 (input layer).

Previous works on layer fusion assume that all the required parameters and the feature pixels with the size of at least $\sum_{l=0}^{n-1} C_{\text {out }}^{(l)} \cdot \mathcal{R}_{h}(l, 1) \cdot \mathcal{R}_{w}(l, 1)+1$ in total must be kept in the on-chip buffer at the same time, only to generate one output pixel for layer-n. For example, all orange

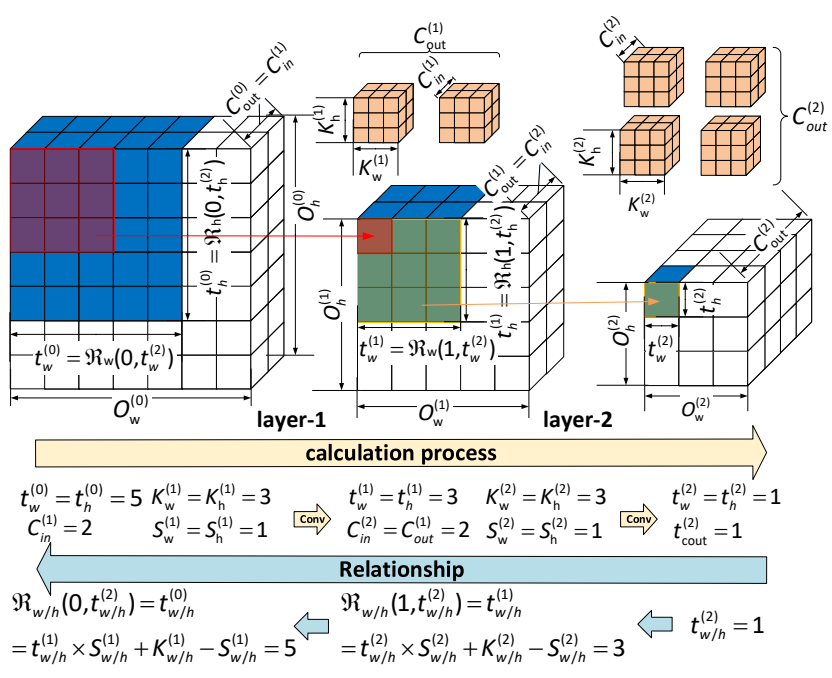

Figure 3. Processing of a fused layer-group.

data (parameters) and at least all blue data (feature pixels) in Fig. 3 must stay in the on-chip buffer at the same time. At this point, the parameters are loaded and kept on-chip, until the computation of the entire fused layergroup has been completed. Thereby, as assumed in prior works, the off-chip memory access volume equals to the sum of the input fmap size of layer-1, the output fmap size of layer- $n$ and the parameter size of the whole layer-group, i.e., $\mid$ ifmap $^{(1)}|+| o \operatorname{fmap}^{(n)}\left|+\sum_{l=1}^{n}\right|$ param $^{(l)} \mid$. Otherwise, this fused-group is deemed invalid due to the lack of on-chip memory space.

However, allowing parameter-induced memory access during the computation of a fused-layer group will possibly gain benefits in terms of the total memory access overhead, since it is very common that the buffer space of a given off-the-shelf DNN processor cannot accommodate all the parameters of the fused layer-group. In addition, allowing parameter refill during the inference process of the fused layer-group exposes the potential benefit of reducing the on-chip buffer space required by the fmap pixels, which can further reduce off-chip memory accesses. Consequently, in contrast to prior work, we will present a memory-cost model (Section 3.2) to precisely evaluate the achievable minimum memory-traffic achieved by each layer-group, which takes into account the parameter-induced memory accesses.

\subsection{Layer Fusion Space Exploration}

In general, DNN algorithms can be represented as computational directed acyclic graphs (CDAGs) $\mathcal{G}=(\mathcal{V}, \mathcal{E})$, which provide a global view of the interconnected layers, but they do not specify how each layer must be implemented [5] as shown in Fig. 1. In CDAGs, nodes $\mathcal{V}$ represent tensor operators (e.g., Conv layer), and edges $\mathcal{E}$ represent data dependencies between layers. Since simple element-wise layers, e.g., batch-normalization $(\mathrm{BN})$ and ReLU, can be directly 


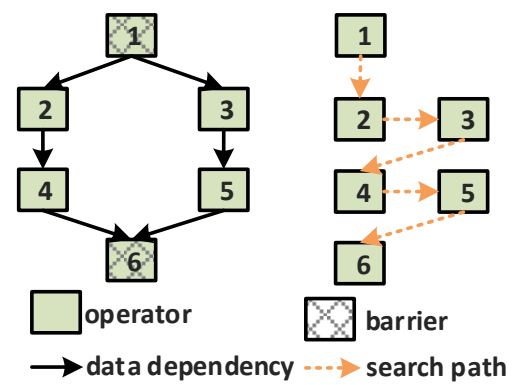

Figure 4. A typical layer fusion search process in prior works.

fused, e.g., Conv+BN+ReLU, they are ignored in the figure for simplicity. In CDAGs, nodes (layers) can be combined into different groups. The various combinations, which constitute the layer fusion search space, lead to different memory overhead. The search space is enormous, and it has been proven to be a NP-hard problem [20] to explore the entire layer fusion space for a complicated NN model.

Fused-layer[1] exhaustively evaluated layer fusion options on their specific hardware design and seek to find the optimal one for simple DNN models without branches, however such an enumeration policy is no longer practical as the network model becomes larger and more complex. DNNVM [30] set the layers that are dependent on more than one layer or by different layers as barriers and assume that a group of layers with barriers in it can be early-rejected during the search of optimal fused-layers, as shown in Fig. 4 (a). However, we found the fusion of layers including barriers can also bring benefits, especially for the network with many branches, e.g., SqueezeNet [16], GoogleNet [26], and NasNet [40]. Thus the results obtained by DNNVM are far from globally optimal. [37] reduced the exploration problem by transforming the complicated network into a linear proxy model without violating the data dependencies, for example, converting the models in Fig. 4 (a) to the linear structure in Fig. 4 (b). Then they applied DP algorithm on the linear structure to find the optimal layer fusion result. However, the result may not be optimal for the original complicated network model, for example, the fused layer-groups $\{2,4\}$ and $\{3,5\}$ will never be identified as a premier solution by this method. Besides, building the linear structure also requires a non-negligible time overhead.

In this work, we put forward an Computing-Graph based off-line hardware-aware layer fusion algorithm (HaFS in Section 3.3) for the original complicated CDAGs of DNNs, which searches through the entire layer fusion space and it is even faster than previous works (Section 4.9). Furthermore, we present a fast on-line layer fusion algorithm (OnFS in Section 3.4) for the online scenario while achieving nearoptimal fusion scheme.

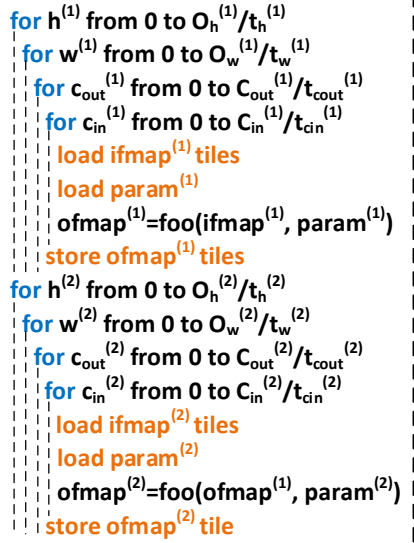

(a)

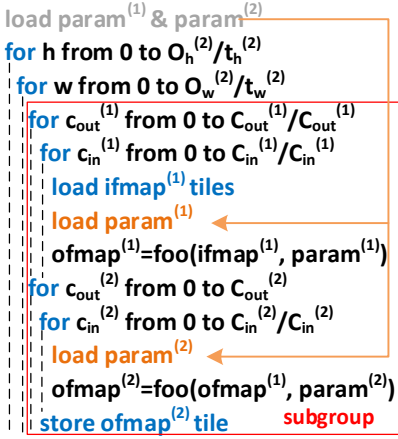

(b)
Figure 5. Pseudo code of the two layers (a) before and (b) after fusing as Fig. 3 (foo represents the execution of the target DNN processor).

\section{Optimal Layer Fusion}

As discussed in prior works [30,37], layer fusion is a general technique to reduce the feature-map induced memory access for DNN processors. In fact, layer fusion only changes the order of operations in the DNN model, and it can be applied to most of the general DNN processors $[4,6-9,18]$. In this section, we introduce the optimal layer fusion framework, Optimus. The layer fusion schemes given by the Optimus could be practiced and applied in the compilation or network-mapping stage to generate the according execution bitstreams or instructions, which will control the hardware to run the network layers in the corresponding dataflow.

\subsection{The Formulation of the Layer-Fusion Problem}

Before jumping to the design of optimal network fuser, we must formalize the network fusion problem, which is not formally or comprehensively defined in prior works. The formulation will guide us to exhaustively explore the entire scheduling space and evaluate the best solution. For a CDAG $\mathcal{G}=(\mathcal{V}, \mathcal{E})$ of a DNN model, let $L=\left\{G_{1}, G_{2}, \ldots\right\}(L \in \mathcal{L}$, $\mathcal{L}$ is the layer fusion space) be a layer fusion scheme that divides the network $\mathcal{V}$ into disjoint fused layer-groups $G_{i}$ $\left(\mathcal{V}=\bigcup G_{i}\right)$ which have no cyclic dependencies in them, as shown in Fig. 1. Thus the optimal layer fusion problem is to find an optimal layer fusion scheme that has the minimum off-chip memory accesses:

$$
\min _{L \in \mathcal{L}} \sum_{G_{i} \in L} \operatorname{MinCost}\left(G_{i}\right)
$$

where MinCost (.) is the cost function that models the memory overhead of the fused layer-groups, which will be detailed in Section 3.2.

\subsection{Cost Estimation of Fused Layer-Groups}

Prior work on layer fusion usually put some restrictions on the configuration of the on-chip memory, so they cannot 
reach the true memory cost of the grouped layers in a network. In this section, we will remodel the memory cost by removing such assumptions.

Allowing parameters-induced access: First of all, unlike prior works, we discard their assumption that all the parameters associated to the fused layers must be kept in the on-chip memory, and then present how to find the true minimum off-chip memory access number for layer-group $G_{i}$ with $n$ layers. As illustrated in Fig. 5, when the on-chip buffer of the processor is large enough to keep all the parameters of the fused layer-group, parameters are loaded outside the loop nest. However, when the parameters of the fused layer-group cannot fit into the on-chip buffer of the target processors, all parameters are loaded in each sub-group iteration. In this case, the parameters in the loop nest do not need to be loaded onto the chip all at once. Instead, only the part of parameters required by the operations are loaded according to the dataflow of the target DNN processors, and the loaded parameters are reused as much as possible by the fmap tiles before releasing the buffer space they occupy, which means the on-chip buffer footprint required by the fused layers are over-estimated in prior works. In our cost model, such a reuse mechanism in the sub-groups are faithfully accounted for. Therefore, we have that the total number of subgroups is $Q=\left\lceil\frac{O_{h}^{(n)}}{t_{h}^{(n)}}\right\rceil \cdot\left\lceil\frac{O_{w}^{(n)}}{t_{w}^{(n)}}\right\rceil$. Accordingly in our cost model, the involved off-chip parameterinduced memory traffic for the layer-group is measured as $p \_a c c e s s=Q \sum_{l=1}^{n} \mid$ param $^{(l)} \mid$. Thus, minimizing $p \_a c c e s s$ is equivalent to maximizing $t_{h}^{(n)} \cdot t_{w}^{(n)}$, which is limited by the on-chip buffer size of a given processor.

Reducing the buffer space requirement: Since the memory cost is directly correlated to the on-chip memory space, we further present how we reduce the on-chip buffer space requirement to the greatest extent for the fmap pixels to approach the true memory lower-bound. In a fused layer-group, we allow the subsequent layer to be invoked immediately once a subset of fmap tiles produced by the predecessor layer are ready. For example, in Fig. 6, layer-1 starts processing $T_{c}$ ifmap tiles as soon as they are provided, which means a buffer space of $T_{c}{ }^{2}$ is sufficient for processing the tiles smoothly.

Although the on-chip buffer space requirement for the fmap pixels can be reduced significantly, the mentioned buffer requirement reduction (BRR) method cannot be applied to two successive layers simultaneously, because it assumes that all channels of ifmap must be reduced to produce one channel of ofmap. As the example illustrated in

\footnotetext{
${ }^{2}$ Herein, $T_{*}$ is the throughput of the processors determined by the PE-array size and the dataflow $[8,31]$, since we realized that there are impacts of tile size on the on-chip resources utilization of the target processor (Section 4.3). It implies that the tile size needs to be greater than the throughput determined by the dataflow and PE-array of a given DNN processor; otherwise, the processor datapath and the corresponding on-chip resources cannot be fully utilized.
}

Fig. 6, buffering only $T_{c}$ ifmap and ofmap tiles of layer-1 causes the partial sums to be evicted to the off-chip memory and then reloaded to the on-chip buffer again as required by the next accumulation operation. In order to deduce the minimum on-chip buffer requirement of the fmap pixels, we present an algorithm to determine which layers in the fused layer-group are compatible to the BRR method.

As formulated in Algorithm 1, the layer group is traversed in reversed topological order (line 1), and we define $d p[l][1]$ as the case when layer- $l$ adopts the BRR method, and $d p[l][0]$ as the case when BRR does not apply. If the layer- $l$ adopts the BRR method, its successor layers must not use the BRR method (line 5); otherwise, its successor layers are allowed to use the BRR method (line 6). Then, we have the BRR decision $\phi(\cdot)$ for each layer and so obtain the minimum on-chip buffer requirement (line 7-8).

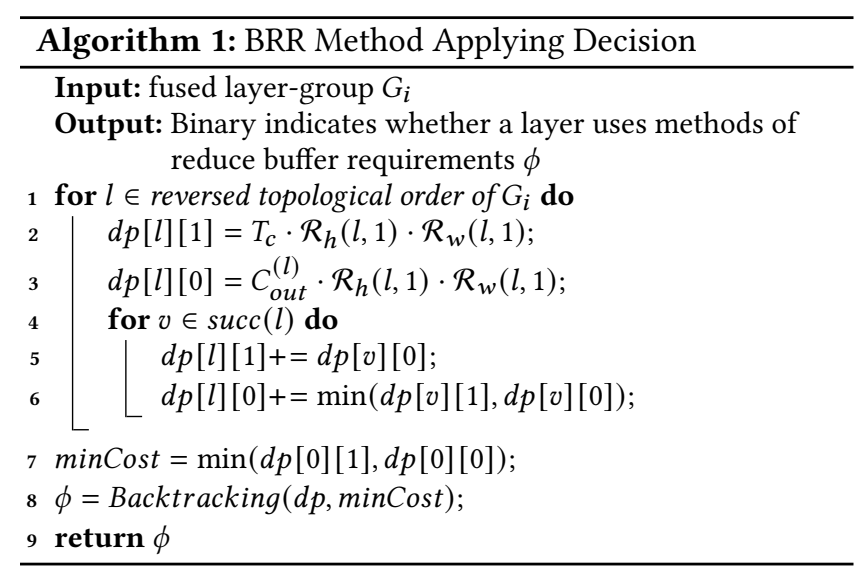

Minimizing off-chip memory access: Having removing the prior memory restrictions, we can maximize $t_{h}^{(n)} \cdot t_{w}^{(n)}$ under the premise that the buffer requirement of fmaps cannot exceed the on-chip buffer capacity $B_{c}$ :

$$
\begin{aligned}
& \max T=\max t_{h}^{(n)} \cdot t_{w}^{(n)} \\
& \text { s.t. } \sum_{l=0}^{n} t_{c}^{(l)} \cdot \mathcal{R}_{h}\left(l, t_{h}^{(n)}\right) \cdot \mathcal{R}_{w}\left(l, t_{w}^{(n)}\right) \leq B_{c} \\
& t_{c}^{(l)}= \begin{cases}\min \left(T_{c}, C_{\text {out }}^{(l)}\right), & \phi(l)=1 \\
C_{\text {out }}^{(l)}, & \phi(l)=0\end{cases} \\
& \quad \min \left(T_{h}, O_{h}^{(n)}\right), \min \left(T_{w}, O_{w}^{(n)}\right) \leq t_{h}^{(n)}, t_{w}^{(n)} \leq O_{h}^{(n)}, O_{w}^{(n)}
\end{aligned}
$$

$t_{c}^{(l)}$ is the channel tile of the ofmap and $\phi(l)$ is the BRR method applying decision from Algorithm 1 . Therefore, the achievable minimum off-chip memory access volume is ${ }^{3}$ :

\footnotetext{
${ }^{3}$ For simple illustration, we assume that there is only one input layer and only one output layer in the layer-group. In fact, our derivation is also applicable to more complicated layer-group with multiple input and output layers, e.g., $\{v 1, v 2, v 3, v 4\}$ in Fig. 7.
} 


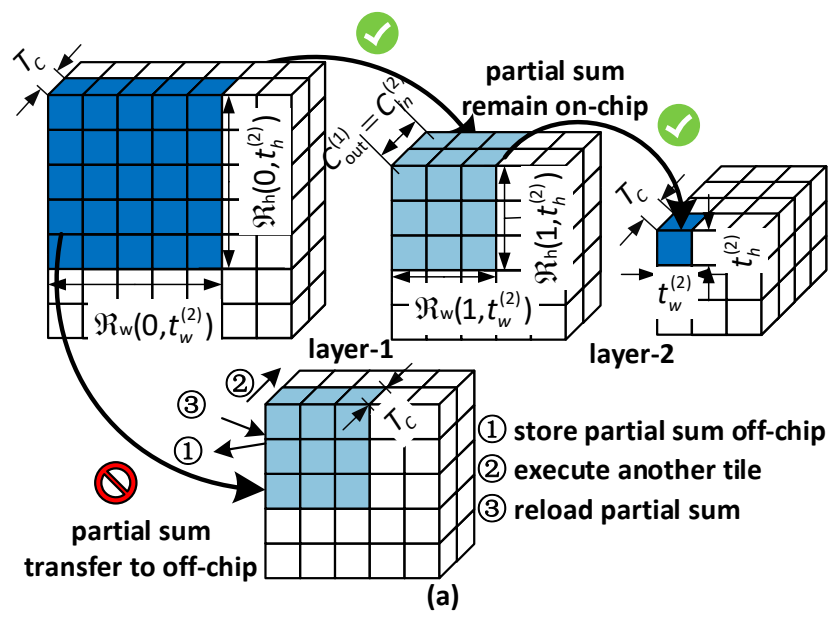

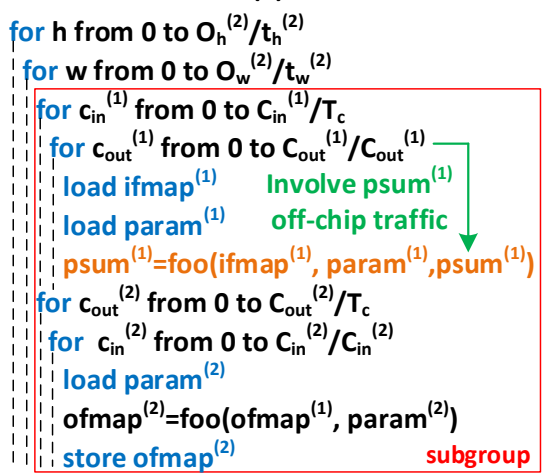

(b)

Figure 6. Reducing the on-chip buffer space requirement of the fused layer-group in Fig. 3.
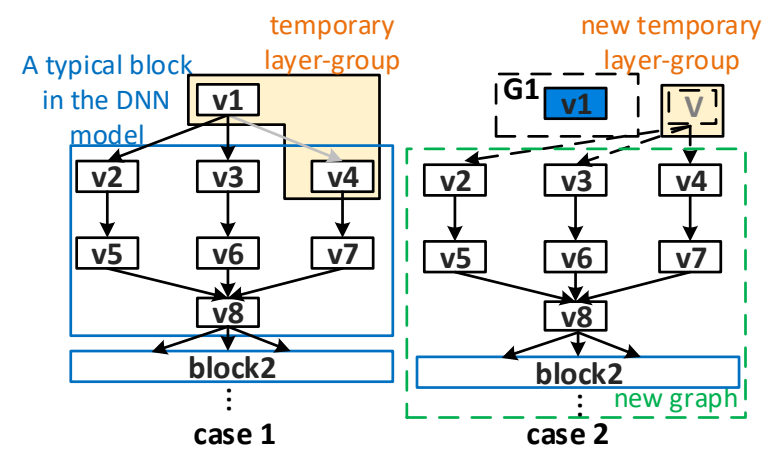

Figure 7. Two cases of DP sub-structure.

$$
\begin{aligned}
\operatorname{MinCost}\left(G_{i}\right)= & \left|\operatorname{ifmap}^{(1)}\right|+\left|\operatorname{ofmap}^{(n)}\right| \\
& +\frac{O_{h}^{(n)} O_{w}^{(n)}}{\max T} \sum_{l=1}^{n}\left|\operatorname{param}^{(l)}\right|
\end{aligned}
$$

Besides the Conv layer, the cost model can be applied to fully-connected layers, deconvolution layers, dilated convolution layers, etc., by reconfiguring the $S_{h}^{(l)}, S_{w}^{(l)}, O_{w}^{(l)}, O_{h}^{(l)}$, $K_{w}^{(l)}$ and $K_{h}^{(l)}$.

\subsection{Off-Line Hardware-Aware Layer Fusion}

So far, based on the computing graph, we have ensured that the potential layer fusion schemes can be correctly evaluated, and then we need to evaluate each fusion scheme in the massive layer fusion space.

Given a CDAG of NN model $\mathcal{G}=(\mathcal{V}, \mathcal{E}), \mathcal{V}=\left\{v_{1}, \ldots, v_{N}\right\}$, as shown in Fig. 7, we observe that for any profitable layergroup (which can contain one or multiple layers), it can grow by including more nodes on the graph or remain unchanged. These two choices are mutually exclusive, and the decisions can be made by comparing the cost of them. However, for complicated NN models, it will make the decision space grow exponentially because there are $2^{\left|\operatorname{succ}\left(v_{i}\right)\right|}$ combination choices for each multi-output node to be included ${ }^{4}$, so the total combination space is $\prod_{i} 2^{\left|\operatorname{succ}\left(v_{i}\right)\right|}$. Fortunately, in layer fusion, layer-groups that result in cyclic data dependencies, e.g., layer-group $\{v 1, v 3, v 6, v 8\}$ in Fig. 7, are invalid; otherwise, the operation on fused layer-groups must be interrupted to load from off-chip memory, e.g., the memory request occurs after $v 1$ to fetch the data from $v 5$ that $v 8$ needs, which stalls the continuous execution of the fused layer-groups. Under this constraint, the total number of available combinations of layer-groups after space growth becomes $\sum_{i} 2^{\left|\operatorname{succ}\left(v_{i}\right)\right|}\left(O\left(2^{\max _{i}\left|\operatorname{succ}\left(v_{i}\right)\right|}|\mathcal{V}|\right)\right)$.

Based on this observation, we present the HaFS algorithm to search for the optimal fusion scheme recursively. We start from the input node $\left(v_{1}\right)$ of $\mathcal{V}$, and a virtual "routing" node of zero memory-overhead will be created as the ancestor node of the original input nodes when there are more than one input nodes. We have a temporary layer-group $\left\{v_{1}\right\}$ that can only be extended but not be reduced, and there are two choices for $\left\{v_{1}\right\}$ as shown in Fig. $7: 1$ ) attach it with one of its external successor node $v_{i}$ if there is no cycle dependencies after graph growth, and the new temporary layer-group becomes $\left.\left\{v_{1}, v_{i}\right\} ; 2\right)$ use it as a valid fused layergroup, i.e., $G_{1}=\left\{v_{1}\right\}$, the new graph becomes $\mathcal{V}=\mathcal{V}-\left\{v_{1}\right\}$, and the temporary layer-group changes accordingly. In both cases, the algorithm works recursively, and thus the original problem is reduced into smaller sub-problems.

By summarizing over these two cases, we can have the general form of sub-problems as: minimizing the memory cost of the graph $V^{\prime}$ which starts from a non-reducible layer-group $G_{t e m p}$. And we can represent the optimal value (minimum memory access number) of the sub-problems as $\mathcal{M}\left(G_{t e m p}, \mathcal{V}^{\prime}\right)$. Therefore, we obtain the optimal substructure property:

$$
\mathcal{M}\left(\left\{v_{1}\right\}, \mathcal{V}\right)=\min \left\{\begin{array}{l}
\min _{v_{i} \in \operatorname{succ}\left(\left\{v_{1}\right\}\right)} \mathcal{M}\left(\left\{v_{1}, v_{i}\right\}, \mathcal{V}\right) \\
\operatorname{MinCost}\left(\left\{v_{1}\right\}\right)+\mathcal{M}\left(\left\{v^{\prime}\right\}, \mathcal{V}-\left\{v_{1}\right\}\right)
\end{array}\right.
$$

\footnotetext{
$\overline{{ }^{4}\left|\operatorname{succ}\left(v_{i}\right)\right|}$ is the number of successor nodes of $v_{i}$.
} 
where $v_{1}=\operatorname{input}(\mathcal{V}), v^{\prime}=\operatorname{input}\left(\mathcal{V}-\left\{v_{1}\right\}\right)$ are the input node of the graph $\mathcal{V}, \mathcal{V}-\left\{v_{1}\right\}$, respectively. $\operatorname{succ}\left(v_{i}\right)$ returns the successor nodes of $v_{i}$.

With the optimal sub-structure property, the HaFS procedure is shown in Algorithm 2. The Fuser first checks the temporary layer-group $G_{t e m p}$, and then initializes it using the input node of the graph (line 2-4) if it is null. Then, Fuser practices the basic steps of DP: 1) Line 5-6 checks whether the sub-problem had already been solved and stored in the global DP table, which implements the "memorizing" mechanism of DP; 2) Line 7-9 returns the result of the base case, i.e., when $G_{t e m p}$ has no successor node. After that, the Fuser extends $G_{t e m p}$ with one of its successor nodes and chooses the optimal one that has minimum off-chip memory traffic (line 13-17) according to the first case of $\mathcal{M}(\cdot)$. Line $14 \mathrm{di}-$ rectly filters the invalid layer-groups that form a cyclic data dependency in the graph. Line 18-23 separates the $G_{t e m p}$ from the other nodes and start again from the remaining graph according to the second case of $\mathcal{M}(\cdot)$. Line 18 avoids the invalid fused layer groups over the virtual nodes. The other layer groups are valid and will be evaluated whether they are profitable or not. Finally, the algorithm returns the optimal layer fusion solutions and the minimum off-chip memory access volume (line 25).

In this way, HaFS explore the whole valid layer fusion space $\mathcal{L}$ and evaluated the memory cost $\sum_{G_{i} \in L} \operatorname{Min} \operatorname{Cost}\left(G_{i}\right)$ of each layer fusion scheme $L=\left\{G_{1}, G_{2}, \ldots\right\}(\forall L \in \mathcal{L})$. The optimal layer fusion scheme and the cost of each sub-graph $\mathcal{V}^{\prime}$ are stored after being evaluated by the memory-cost model, to avoid repetitively solving the same sub-problems.

\subsection{On-Line Hardware-Aware Layer Fusion}

When the chip resources such as on-chip memory are allocated on-line, the decision of layer fusion must be made at a reasonable time overhead in case of penalizing the network inference performance. The complexity of HaFS may add a significant amount of time overhead to the millisecondslevel network inference latency when being executed on-line. Therefore, we need a fast and efficient on-line fusion strategy, OnFS, to deploy fused neural networks at runtime. Intuitively, shrinking the search space of layer fusion schemes can dramatically save the algorithm runtime overhead. Fortunately, we found some empirical rules from experimental results with HaFS.

First, in some cases, there are very limited benefits gained to fuse a layer and only part of its fan-out successor layers into the same group, because the intermediate data still need to be written back to the off-chip memory for the remaining consumers. For example, in the layer-group $\{\mathrm{v} 1, \mathrm{v} 2, \mathrm{v} 3\}$ of Fig. 7, the intermediate data of layer $v 1$ will be transfer to the off-chip memory for the layer $v 4$ to use. Thus, a layer should be either fused with, or separated from, all of its fan-out successor layers.

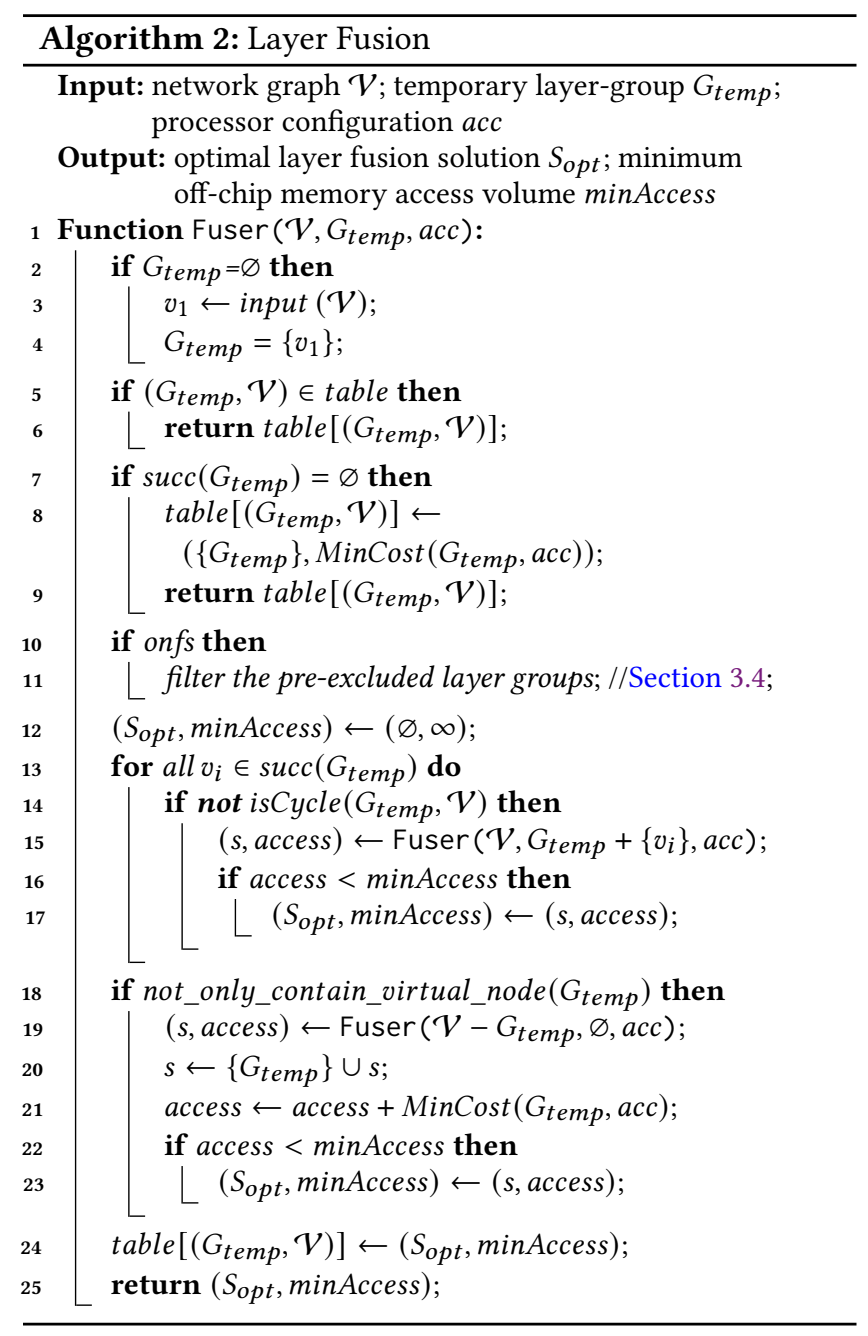

Second, it is not rewarding to fuse the layers into the same group if they share no data dependencies, as the layer $v 5$ and $v 6$ shown in Fig. 7. The data dependencies to exploit can be either due to sharing the same input with another layer in the layer-group, or consuming the output data of a layer in the layer-group. For example, $v 3$ shares input data with $\mathrm{v} 2$ and thus the layer-group $\{\mathrm{v} 2, \mathrm{v} 3\}$ is profitable. Thus, we see that a layer can be added into a temporary layer-group only if it shares some data dependencies with other layers in the layer-group.

Third, according to the equation (2) and (3), the more layers are fused, the more feature-induced memory accesses are reduced, but it will introduce excessive parameters-induced memory accesses. Therefore, we can reduce the search space by limiting the size of the layer-group and avoiding too many parameter-induced memory accesses. However, the proper layer-group size limitation varies with the on-chip memory size. Consequently, we devise a look-up table mechanism to quickly determine the layer-group size limitation for each dynamically allocated on-chip memory size. 
The look-up table is pre-built in the Algorithm 3. Each table entry corresponds to the on-chip memory size memo $[n]$, and the infeasible layer-group size $n$ associated to this entry, wherein memo $[n]=\min _{\left|G_{i}\right|=n} \sum_{l=0}^{n} t_{c}^{(l)} \mathcal{R}\left(l, t_{h}^{(n)}\right) \mathcal{R}\left(l, t_{w}^{(n)}\right)$ and $t_{c}^{(l)}$ determined by Algorithm 1 . And $t_{h}^{(n)}$ and $t_{w}^{(n)}$ cannot be too small, otherwise the memory access induced by the parameter $p \_a c c e s s=\left[\frac{O_{h}^{(n)} O_{w}^{(n)}}{t_{h}^{(n)} t_{w}^{(n)}}\right] \sum_{l=1}^{n} \mid$ param $^{(l)} \mid$ is too large. According to a large number of experiments and analyses, we conclude that if the volume of the access is 16 times the number of parameters, the loss is possibly greater than the gain, thus the $t_{h}^{(n)}$ and $t_{w}^{(n)}$ are set to $O_{h}^{(n)} / 4$ and $O_{w}^{(n)} / 4$ (almost all networks have $\left.O_{h}^{(n)}=O_{w}^{(n)}\right)$. Therefore, memo $[n]$ is a minimum memory size to ensure the benefit of the layergroup whose size does not exceed $n$, and memo can be proved to be increasing.

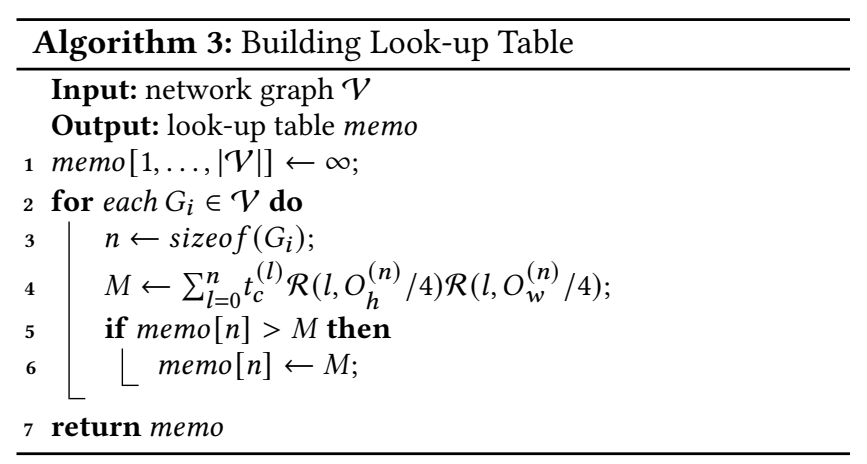

With this compact look-up table, when given the buffer capacity $B_{c}$ online, we can quickly estimate the maximum group size it allows, and use it to filter many of the fusion options. That is, if the target network is allocated with an onchip memory capacity $B_{c}$ in between memo $[n]$ and memo $[n+$ 1] in the table, i.e. memo $[n]<B_{c}<\operatorname{memo}[n+1]$, the grouplayers with size larger than $n$ can be rejected early without being evaluated.

By imposing the above three rules we observed from fusion scheme exploration, the search space of OnFS is significantly pruned, and it is a heuristic algorithm designed based on HaFS. If OnFS mode is set to true, the fused layer-groups that do not satisfy these three rules are excluded in line 11 of Algorithm 2.

\section{Evaluation}

\subsection{Experiment Setup}

Workloads: We evaluate Optimus using several state-ofthe-art CNN models, including the classic AlexNet [19], VGG16 [25], GoogleNet [26], SqueezeNet [16], MobileNet [15], ResNet [14], and the latest NasNet designed via NAS [40]. Unless otherwise specified, the batch size of these workloads is set to 4 .
Table 1. DNN Processor Configurations

\begin{tabular}{l|l}
\hline on-chip buffer & $128 \mathrm{~KB}$ \\
PE-array & $32 \times 32$ \\
register files/PE & $64 \mathrm{~B}$ \\
dataflow & $O_{w} \mid C_{\text {out }}^{*}$ \\
arithmetic units & 16 -bit fixed-point \\
\hline${ }^{*}$ parallel the loop $O_{w}$ and $C_{\text {out }}[31]$
\end{tabular}

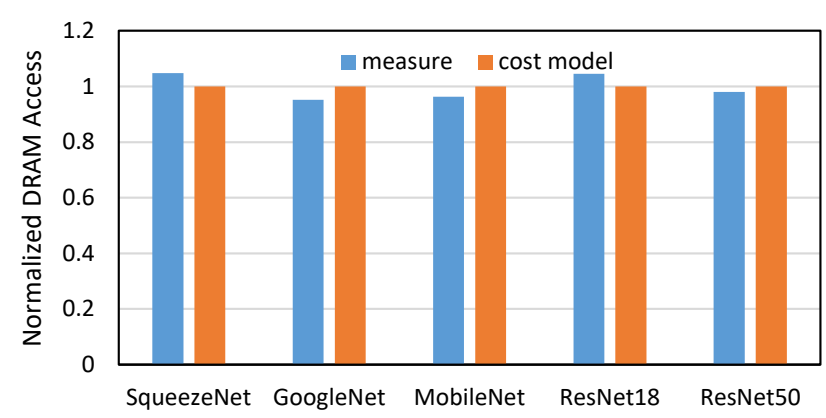

Figure 8. Accuracy of the memory model against measure results.

Baselines: To fairly evaluate Optimus, we use the latest network fusers, FusedLayer [1], DNNVM [30] and Efficient-S [37] as the baselines. FusedLayer is designed to fuse the adjacent neural layers of the models without branches, thus, it is applied to VGG16 and AlexNet only. To evaluate the effectiveness of our approach for DNN processors of different dataflow and architectures, we apply it to the design of ShiDianNao [9], Eyeriss [8] and [6].

Hardware setup: In evaluation, Optimus and the baselines are applied to the DNN processors as in Fig. 2, which are implemented and synthesized with Design Compiler using $65 \mathrm{~nm}$ process technology. Unless otherwise specified, the DNN processor configurations are shown in Table 1 . We also use the analysis framework as [8] to estimate the performance and the energy efficiency of the DNN processors for rapid performance analysis, including the energy cost for data accesses from DRAM, on-chip buffer (SRAM), PE-array (inter-PE communication), and RFs (register files) and the energy cost of MACs. CACTI 7.0 [2] is used to simulate both the SRAM buffer and the DRAM (DDR3-800).

\subsection{Accuracy of the Memory Model}

We thoroughly validate the accuracy of the memory cost model by comparing its results to our complete DNN processor design generated by the synthesis toolchain. Fig. 8 shows the DRAM access comparison between the cost model and the measure results and the errors are less than $5 \%$.

\subsection{Performance and Energy Comparison}

Fig. 9 shows the energy comparison over all workloads between the baselines and Optimus. Compared to DNNVM and 
Efficient-S, HaFS obtains $1.73 \mathrm{x}-3.94 \mathrm{x}$ and $1.71 \mathrm{x}-3.09 \mathrm{x}$ energy efficiency (except ALU energy) respectively, while OnFS achieves up to $3.74 \mathrm{x}$ and $2.86 \mathrm{x}$ energy efficiency, respectively.

The baselines do not consider the influence of processor architectures, such as the PE-array size and the dataflow type, on the efficacy of layer fusion options [1, 30, 37]. Consequently, they cannot well exploit the reusability of data in the low-cost PE local RFs and inter-PE communication when fusing the layers, so that they induce relatively more frequent accesses to the on-chip buffer and higher on-chip buffer energy cost. In contrast, Optimus is fully aware of the processors architectures in Section 3.2, thus it will not deteriorate the energy efficiency of other components while reducing the energy cost of DRAM.

Fig. 9 also shows the end-to-end latency comparison between Optimus and the baselines. Optimus achieves significant performance gains when the DRAM cost dominates the network inference latency. Compared with DNNVM and Efficient-S, it shows an average improvement of $1.1 \mathrm{x}$ and $1.4 \mathrm{x}$, respectively.

\subsection{Memory-Level Analysis}

As shown in Table 2, compared to DNNVM and Efficient-S, HaFS reduces reduces $16.8 \%-72.2 \%$ and $15.0 \%$ - 52.4\% DRAM accesses, respectively, while OnFS (not listed in the table due to space constraints) reduces $16.3 \%-69.2 \%$ and $10.1 \%$ - $47.2 \%$ DRAM accesses. The improved results vary to the specific benchmarks, because the achievable minimum memory overhead and the optimal layer fusion scheme heavily depend on the workloads. In some network architectures, especially those with a similar structure to SqueezeNet, the performance of prior works on layer fusion is far from optimal.

To further analyze the effectiveness of the optimization techniques proposed in Section 3, Fig. 10 compares the memory cost of HaFS against that of the four alternative implementations which selectively disable one of the optimization options. When comparing HaFS to w/o fusion (only fuses the layers with the simple element-wise operations, e.g., $\mathrm{CONV}+\mathrm{BN}+\mathrm{ReLU}$ ), layer fusion technology reduces the DRAM access significantly. Comparing with w/o MinCost (use the cost function of FusedLayer [1]), HaFS benefits from the memory-cost model MinCost (.) in Section 3.2, because it takes into consideration that the parameters of fused layergroups may not fit into the on-chip buffer and captures the minimum overall off-chip memory access accurately which makes sure the optimal network fuser goes towards the correct search direction. Compared to w/o full space 1 (explore the restricted space of DNNVM in Fig. 4 (a)) and w/o full space2 (explore the restricted space of Efficient-S in Fig. 4 (b)), the CNN models with complicated architecture, such as SqueezeNet, GoogleNet, ResNet and NasNet, benefit from the full exploration of the layer fusion spaces. Efficient-S explores a larger space and achieves better results in SqueezeNet and
ResNet; still, this restricted space is not enough for more complex structures, such as GoogleNet and NasNet.

\subsection{Impact of Batch Size}

Table 3 shows the impact of batch size on the memory-level performance of VGG16 and SqueezeNet. HaFS is significantly superior to DNNVM and Efficient-S under a variety of batch sizes. When the batch size of VGG16 and SqueezeNet is greater than 4, the DRAM access number stops dropping, because increasing the batch size can improve the reuse opportunities of weight only when the batch size is small. However, when the batch size becomes larger, the limited on-chip memory space prevents it from taking advantage of the data reuse opportunities.

\subsection{Impact of On-chip Memory Space}

The analysis in Section 3.2 indicates that the on-chip buffer capacity is an important factor that influences the DRAM access traffic. We test the DRAM access volume of Optimus under different on-chip buffer capacities, as shown in Fig. 11. Oracle refers to the ideal case when all the weights and feature maps of the network are accessed once without any intermediate feature-induced off-chip traffic. It is seen that Optimus outperforms DNNVM and Efficient-S in all cases with different on-chip buffer size. All these solutions under comparison achieve relatively more DRAM access reduction on larger on-chip buffers, while the marginal benefits brought by buffer increases are higher when the on-chip buffer size is small (smaller than $96 \mathrm{~KB}$ ). When the on-chip buffer size is larger than $96 \mathrm{~KB}$, the restricted layer fusion space stops DNNVM from further reducing DRAM accesses, since there is no more layer-fusion potential available from $D N N V M$ to exploit. On the contrary, when buffer size increases, Optimus and Efficient-S still produce performance gains even though at a much lower rate than that in smaller buffers, until all intermediate feature maps can be consumed on-chip and no longer evicted out to the memory. However, in cases of smaller buffers, Efficient-S does not perform as well as Optimus, because its simplified search strategy will omit some profitable layer-groups by default, and it does not have an accurate cost estimation to drive their search algorithm.

\subsection{Impact of Processor Architecture}

In Fig. 12, We can see Optimus helps DNN processors of different dataflow achieve similar DRAM access and energy efficiency on the same hardware configuration since Optimus is hardware-aware and able to locate the optimal layer fusion solutions for different dataflows. Fig. 13 shows the DRAM access number and the energy efficiency of Optimus on different PE-array size and on-chip buffer (KB). According to $\operatorname{MinCost}(\cdot)$, enlarging the PE-array will result in a slight increase in the number of memory requests, but the total 
Table 2. Statistic on DRAM accesses (MB) of Optimus and the baselines.

\begin{tabular}{lccccccccc}
\hline & VGG16 & AlexNet & SqueezeNet & GoogleNet & MobileNet & ResNet18 & ResNet50 & ResNet152 & NasNet(large) \\
\hline HaFS & 333 & 15 & 20 & 56 & 33 & 58 & 226 & 602 & 4522 \\
\hline Efficient-S & $476(-30.1 \%)$ & $18(-16.8 \%)$ & $42(-52.4 \%)$ & $78(-28.0 \%)$ & $41(-20.1 \%)$ & $68(-15.0 \%)$ & $280(-19.3 \%)$ & $832(-27.7 \%)$ & $5435(-16.8 \%)$ \\
\hline DNNVM & $476(-30.1 \%)$ & $18(-16.8 \%)$ & $71(-72.2 \%)$ & $92(-39.1 \%)$ & $41(-20.1 \%)$ & $85(-31.8 \%)$ & $292(-22.7 \%)$ & $841(-28.5 \%)$ & $5695(-20.6 \%)$ \\
\hline
\end{tabular}

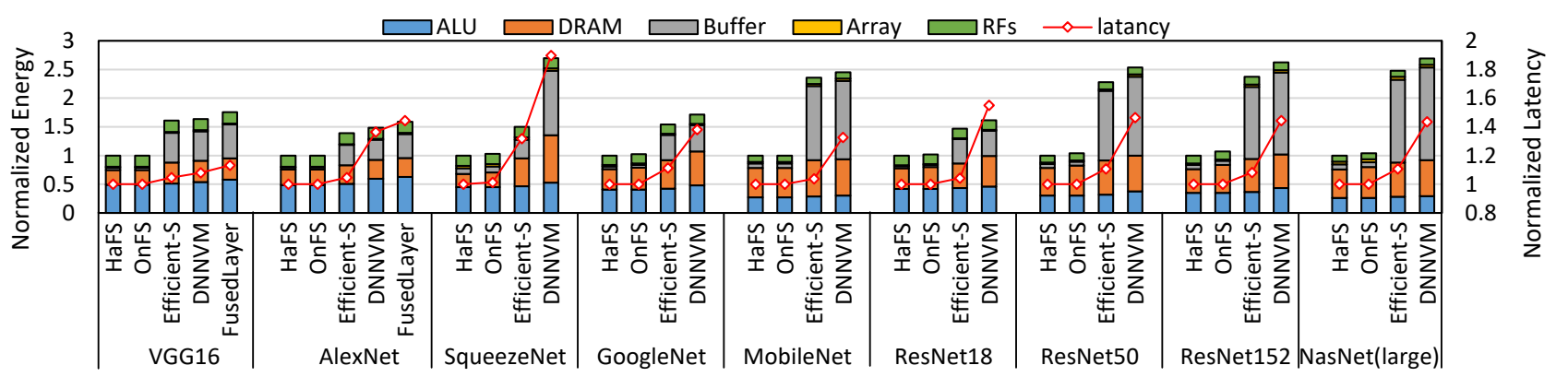

Figure 9. Performance and energy comparison between baselines and Optimus.

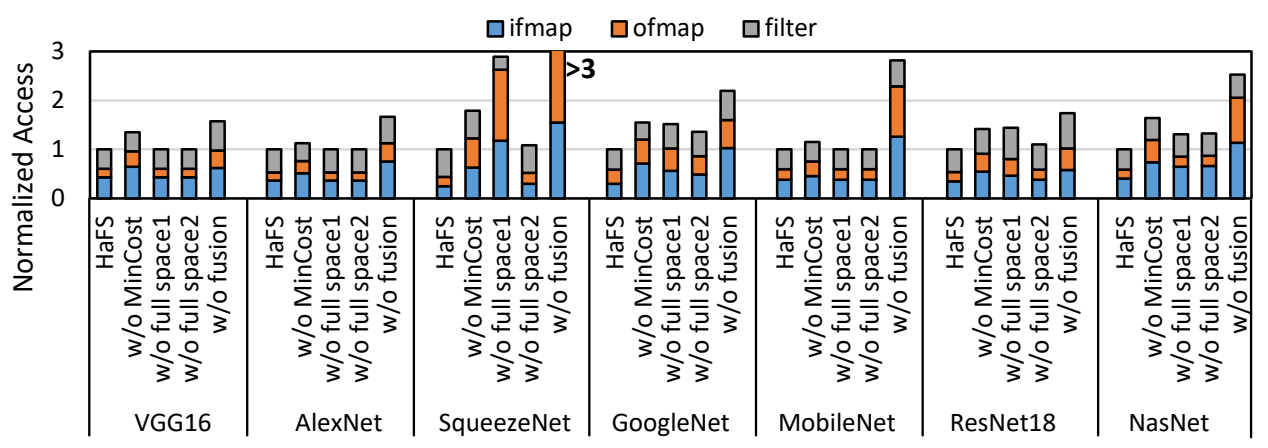

Figure 10. Memory-level analysis.

Table 3. Impact of Batch Size (1e-3 DRAM Access /MAC)

\begin{tabular}{c|cccccc|cccccc}
\hline method & \multicolumn{7}{|c|}{ VGG16 } & \multicolumn{5}{c}{ SqueezeNet } \\
\hline batch & 1 & 2 & 4 & 8 & 16 & 64 & 1 & 2 & 4 & 8 & 16 & 64 \\
\hline HaFS & 2.7 & 2.5 & 2.4 & 2.4 & 2.4 & 2.4 & 3.4 & 3.2 & 3.1 & 3.1 & 3.1 & 3.1 \\
DNNVM & 3.5 & 3.3 & 3.2 & 3.2 & 3.2 & 3.2 & 9.5 & 9.3 & 9.3 & 9.3 & 9.3 & 9.3 \\
Efficient-S & 3.5 & 3.3 & 3.2 & 3.2 & 3.2 & 3.2 & 5.7 & 5.4 & 5.3 & 5.3 & 5.3 & 5.3 \\
\hline
\end{tabular}

energy consumption will decrease due to higher computation efficiency and shortened latency. In general, the optimal layer fusion solution is varying to the processor configurations, and the Optimus is generalizable to different processor architectures.

\subsection{Performance on Different Processors}

Optimus can easily be applied to most of the deep learning processors and minimize their off-chip memory accesses. As a case study, we integrate Optimus into three DNN processors, ShiDianNao, Eyeriss and [6], as shown in Table 4. Note that the buffer of ShiDianNao is split into three units: an input buffer, an output buffer and a synapse buffer. [6] is designed to achieve the communication lower bound for the Conv layers, but do not consider the optimization of layer fusion. Optimus even achieves better results on Eyeriss without using data compression when compared to baseline Eyeriss with sparse compression, and it outperforms the baseline ShiDianNao when applied to the ShiDianNao accelerator. Optimus also further drops the off-chip communication lower bound for the convolutional accelerator proposed in [6], because Optimus can eliminate the unnecessary off-chip traffic induced by intermediate feature maps.

Table 4. Reduction in memory access (\%) compared to the processor baselines

\begin{tabular}{l|ccccc}
\hline & AlexNet & VGG16 & GoogleNet & ResNet18 & SqueezeNet \\
\hline Eyeriss & 33.25 & 31.75 & 49.56 & 34.44 & 87.59 \\
ShiDianNao & 37.86 & 52.45 & 49.45 & 24.39 & 85.26 \\
[6] & 32.43 & 27.41 & 47.31 & 16.86 & 84.29 \\
\hline
\end{tabular}

\subsection{Algorithm Overhead Analysis}

Table 5 presents the number of evaluated layer-group options and the time overhead it takes by the algorithms to search for the final fusion solutions. The experiment is conducted on Inter(R) Core(TM) i7-6700 CPU @ 3.40GHz, and the OS is Ubuntu 16.04.4 LTS. It shows that the runtime overhead of Optimus is within a reasonable range. For example, the HaFS program takes $0.36-3.28 \mathrm{e}+05 \mathrm{~ms}$ to generate the optimal layer fusion solutions which is also much faster than 
Table 5. Execution time of layer fusion algorithm

\begin{tabular}{l|c|c|cc|cc}
\hline \multirow{2}{*}{ workload } & \multirow{2}{*}{ max\# } & \multirow{2}{*}{$\#$} & \multicolumn{2}{|c|}{ \#groups } & \multicolumn{2}{c}{ latency(ms) } \\
\cline { 4 - 7 } & $\mid$ succ $\mid$ & layers & HaFS & OnFS & HaFS & OnFS \\
\hline VGG & 1 & 18 & 171 & 80 & 0.36 & 0.18 \\
ResNet50 & 2 & 52 & 3510 & 195 & 14.59 & 0.64 \\
ResNet152 & 2 & 154 & 24250 & 467 & 173.43 & 3.22 \\
GoogleNet & 4 & 72 & 50436 & 423 & 514.53 & 3.43 \\
SqueezeNet & 2 & 38 & 1057 & 216 & 3.39 & 0.47 \\
NasNet & 10 & 456 & $2.9 \mathrm{e}+07$ & 1627 & $3.28 \mathrm{e}+05$ & 45.38 \\
\hline
\end{tabular}

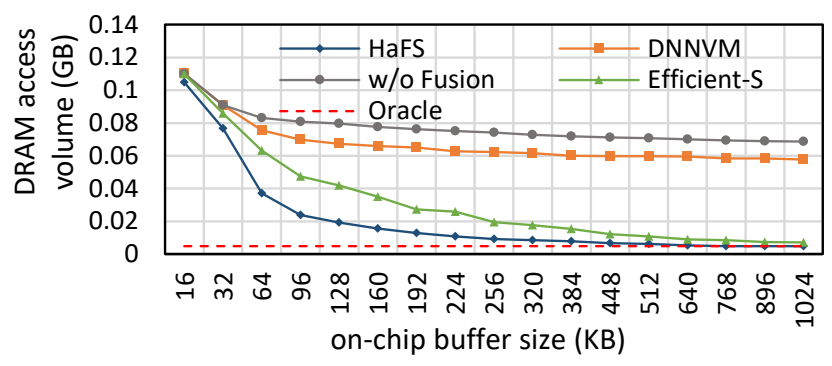

Figure 11. Different on-chip buffer capacities (SqueezeNet).

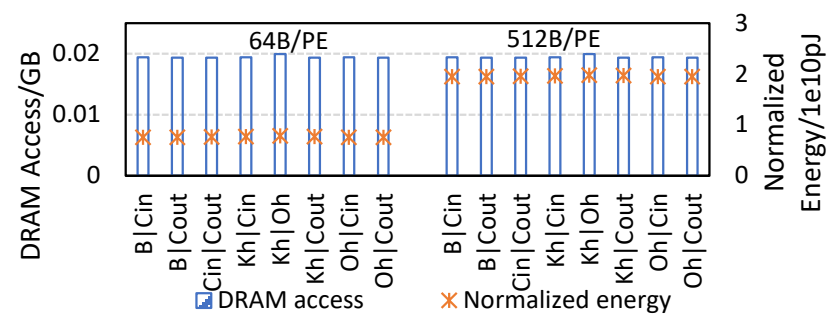

Figure 12. Different dataflow (SqueezeNet).

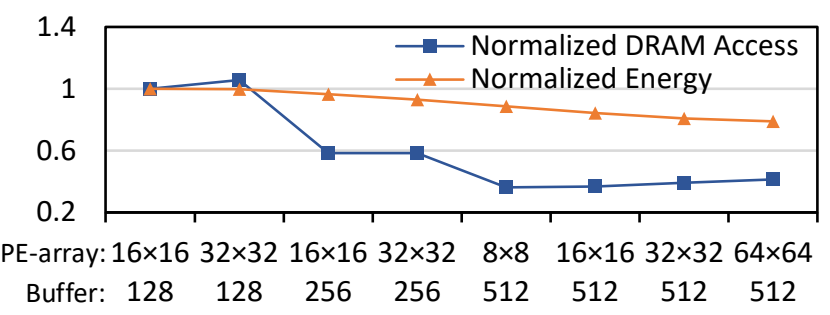

Figure 13. The impact of PE-array size (SqueezeNet).

what has been reported in previous work [17, 30, 37]. In contrast, OnFS costs only $0.18-45.38 \mathrm{~ms}$ to achieve near-optimal fusion schemes online.

\section{Related Work}

State-of-the-art DNN processors: To overcome the computing challenge of DNNs, lots of specialized ASIC-based [4, 7-9] and FPGA-based [1, 12, 24, 28, 29, 32, 34] DNN processors have been proposed for better performance or energyefficiency. [6, 8, 9, 12, 24], etc., process networks layer by layer, targeting at reduce the data transfer incurred for each layer individually. [1, 27, 29] instantiate a fusion design for dedicated NN models on FPGA, however, it is difficult to scale these designs for deeper and more complicated networks, and re-configuration overheads are introduced when switching to other models. Sparse DNN accelerators, e.g., [11-13, 23, 33, 35, 36], improved NN efficiency by avoiding redundant operations and reducing memory footprints, which are orthogonal to our exploration.

Layer fusion technique: [21, 22], etc., introduced fusion methods to various computer vision and computational photography algorithms for general-purpose processors, e.g., CPU and GPU. [17] directly restricted the number of nodes in a single group to reduce the fusion searching efforts, exploring more potentially profitable fusion opportunities compared with [21, 22]. Their target algorithms perform 1D or 2D convolution, rather than the 3D convolutions needed for DNNs. Besides, focusing on specialized processor allows us to use a large amount of information to model the achievable minimum memory overhead of the fused layer-groups.

On DNN processors, [29, 38] use DP algorithm to find the optimal layer fusion option for simple DNN models without branches unlike the straightforward enumeration-andevaluate strategy as in [1]. [30,37] attempt to explore the restricted layer fusion space for the complicated networks. [37] proposed backtracking-based approach to determine the execution order within a fused layer-group, releasing the buffer occupation as soon as possible, and [29] proposed line buffer technique to avoid introducing additional buffer resource consumption or computational redundancy for overlapping input data in layer fusion, which are orthogonal to our work. To the best of our knowledge, we are the first work to give the achievable minimum memory-cost model for fused layer-groups and also the first work to discuss how to reach fast and high-performance online layer fusion.

\section{Conclusion}

In this paper, we redefine the layer-fusion problem for neural network applications, and propose Optimus, a novel neural network fusion framework to achieve the optimal layer fusion for arbitrary network architectures and different stateof-the-art DNN processors. Optimus includes an off-line and an on-line layer fusion algorithm, and an accurate memory cost function that directs the search procedure towards the memory-optimal layer fusion solution. In evaluation with state-of-the-art workloads and DNN accelerator implementations, the experiment results demonstrate that Optimus reduces $10.1 \%-72.2 \%$ off-chip memory accesses, obtains up to $3.94 \mathrm{x}$ energy efficiency over the baselines on processors of different architectures and dataflows.

\section{Acknowledgments}

This work was supported by the National Natural Science Foundation of China (under Grants No. 61876173), and the Strategic Priority Research Program of Chinese Academy of Science (under Grant No. XDC05030201). 


\section{References}

[1] Manoj Alwani, Han Chen, Michael Ferdman, and Peter Milder. 2016. Fused-layer CNN accelerators. In Proceedings of the Annual IEEE/ACM International Symposium on Microarchitecture (MICRO). IEEE, 1-12. https://doi.org/10.1109/MICRO.2016.7783725

[2] Rajeev Balasubramonian, Andrew B. Kahng, Naveen Muralimanohar, Ali Shafiee, and Vaishnav Srinivas. 2017. CACTI 7: New Tools for Interconnect Exploration in Innovative Off-Chip Memories. ACM Trans. Archit. Code Optim. 14, 2 (2017), 14:1-14:25. https://doi.org/10. 1145/3085572

[3] Luiz André Barroso and Urs Hölzle. 2007. The case for energyproportional computing. Computer 40, 12 (2007), 33-37. https: //doi.org/10.1109/MC.2007.443

[4] Tianshi Chen, Zidong Du, Ninghui Sun, Jia Wang, Chengyong Wu, Yunji Chen, and Olivier Temam. 2014. DianNao: A Small-footprint High-throughput Accelerator for Ubiquitous Machine-learning. In Proceedings of the International Conference on Architectural Cupport for Programming Languages and Operating Systems (ASPLOS). 269-284. https://doi.org/10.1145/2541940.2541967

[5] Tianqi Chen, Thierry Moreau, Ziheng Jiang, Lianmin Zheng, Eddie Yan, Haichen Shen, Meghan Cowan, Leyuan Wang, Yuwei Hu, Luis Ceze, et al. 2018. \{TVM $\}$ : An automated end-to-end optimizing compiler for deep learning. In 13th \{USENIX\} Symposium on Operating Systems Design and Implementation (\{OSDI\} 18). 578-594. https://doi.org/10. 5555/3291168.3291211

[6] Xiaoming Chen, Yinhe Han, and Yu Wang. 2020. Communication Lower Bound in Convolution Accelerators. In 2020 IEEE International Symposium on High Performance Computer Architecture (HPCA). IEEE, 529-541. https://doi.org/10.1109/HPCA47549.2020.00050

[7] Yunji Chen, Tao Luo, Shaoli Liu, Shijin Zhang, Liqiang He, Jia Wang, Ling Li, Tianshi Chen, Zhiwei Xu, Ninghui Sun, et al. 2014. Dadiannao: A machine-learning supercomputer. In Proceedings of the Annual IEEE/ACM International Symposium on Microarchitecture (MICRO). IEEE, 609-622. https://doi.org/10.1109/MICRO.2014.58

[8] Yu-Hsin Chen, Joel Emer, and Vivienne Sze. 2016. Eyeriss: a spatial architecture for energy-efficient dataflow for convolutional neural networks. In Proceedings of the International Symposium on Computer Architecture (ISCA). 367-379. https://doi.org/10.1145/3007787.3001177

[9] Zidong Du, Robert Fasthuber, Tianshi Chen, Paolo Ienne, Ling Li, Tao Luo, Xiaobing Feng, Yunji Chen, and Olivier Temam. 2015. ShiDianNao: Shifting vision processing closer to the sensor. In Proceedings of the Annual International Symposium on Computer Architecture (ISCA). 92104. https://doi.org/10.1145/2749469.2750389

[10] Mingyu Gao, Xuan Yang, Jing Pu, Mark Horowitz, and Christos Kozyrakis. 2019. Tangram: Optimized coarse-grained dataflow for scalable NN accelerators. In Proceedings of the International Conference on Architectural Support for Programming Languages and Operating Systems (ASPLOS). 807-820. https://doi.org/10.1145/3297858.3304014

[11] Ashish Gondimalla, Noah Chesnut, Mithuna Thottethodi, and TN Vijaykumar. 2019. SparTen: A Sparse Tensor Accelerator for Convolutional Neural Networks. In Proceedings of the International Symposium on Microarchitecture (MICRO). 151-165. https://doi.org/10.1145/ 3352460.3358291

[12] Song Han, Junlong Kang, Huizi Mao, Yiming Hu, Xin Li, Yubin Li, Dongliang Xie, Hong Luo, Song Yao, Yu Wang, et al. 2017. Ese: Efficient speech recognition engine with sparse lstm on fpga. In Proceedings of the ACM/SIGDA International Symposium on Field-Programmable Gate Arrays (FPGA). 75-84. https://doi.org/10.1145/3020078.3021745

[13] Song Han, Xingyu Liu, Huizi Mao, Jing Pu, Ardavan Pedram, Mark A Horowitz, and William J Dally. 2016. EIE: efficient inference engine on compressed deep neural network. In Proceedings of the International Symposium on Computer Architecture (ISCA). 243-254. https://doi.org/ 10.1109/ISCA.2016.30
[14] Kaiming He, Xiangyu Zhang, Shaoqing Ren, and Jian Sun. 2016. Deep residual learning for image recognition. In Proceedings of the IEEE conference on computer vision and pattern recognition. 770-778. https: //doi.org/10.1109/CVPR.2016.90

[15] Andrew G Howard, Menglong Zhu, Bo Chen, Dmitry Kalenichenko, Weijun Wang, Tobias Weyand, Marco Andreetto, and Hartwig Adam. 2017. Mobilenets: Efficient convolutional neural networks for mobile vision applications. arXiv preprint arXiv:1704.04861 (2017). https: //arxiv.org/abs/1704.04861

[16] Forrest N Iandola, Song Han, Matthew W Moskewicz, Khalid Ashraf, William J Dally, and Kurt Keutzer. 2016. SqueezeNet: AlexNet-level accuracy with 50x fewer parameters and $<0.5 \mathrm{MB}$ model size. arXiv preprint arXiv:1602.07360 (2016). https://arxiv.org/abs/1602.07360

[17] Abhinav Jangda and Uday Bondhugula. 2018. An effective fusion and tile size model for optimizing image processing pipelines. $A C M$ SIGPLANNotices 53, 1 (2018), 261-275. https://doi.org/10.1145/3200691. 3178507

[18] Norman P Jouppi, Cliff Young, Nishant Patil, David Patterson, Gaurav Agrawal, Raminder Bajwa, Sarah Bates, Suresh Bhatia, Nan Boden, Al Borchers, et al. 2017. In-datacenter performance analysis of a tensor processing unit. In Proceedings of the 44th annual international symposium on computer architecture. 1-12. https://doi.org/10.1145/ 3079856.3080246

[19] Alex Krizhevsky, Ilya Sutskever, and Geoffrey E Hinton. 2017. ImageNet classification with deep convolutional neural networks. Commun. ACM 60, 6 (2017), 84-90. https://doi.org/10.1145/3065386

[20] Orlando Moreira, Merten Popp, and Christian Schulz. 2017. Graph partitioning with acyclicity constraints. arXiv preprint arXiv:1704.00705 (2017). https://doi.org/10.4230/LIPIcs.SEA.2017.30

[21] Ravi Teja Mullapudi, Andrew Adams, Dillon Sharlet, Jonathan RaganKelley, and Kayvon Fatahalian. 2016. Automatically scheduling halide image processing pipelines. ACM Transactions on Graphics (TOG) 35 , 4 (2016), 1-11. https://doi.org/10.1145/2897824.2925952

[22] Ravi Teja Mullapudi, Vinay Vasista, and Uday Bondhugula. 2015. Polymage: Automatic optimization for image processing pipelines. ACM SIGARCH Computer Architecture News 43, 1 (2015), 429-443. https://doi.org/10.1145/2694344.2694364

[23] Angshuman Parashar, Minsoo Rhu, Anurag Mukkara, Antonio Puglielli, Rangharajan Venkatesan, Brucek Khailany, Joel Emer, Stephen W Keckler, and William J Dally. 2017. SCNN: An accelerator for compressed-sparse convolutional neural networks. In Proceedings of the Annual International Symposium on Computer Architecture (ISCA). IEEE, 27-40. https://doi.org/10.1145/3079856.3080254

[24] Jiantao Qiu, Jie Wang, Song Yao, Kaiyuan Guo, Boxun Li, Erjin Zhou, Jincheng Yu, Tianqi Tang, Ningyi Xu, Sen Song, et al. 2016. Going deeper with embedded fpga platform for convolutional neural network. In Proceedings of the ACM/SIGDA International Symposium on FieldProgrammable Gate Arrays (FPGA). 26-35. https://doi.org/10.1145/ 2847263.2847265

[25] Karen Simonyan and Andrew Zisserman. 2015. Very Deep Convolutional Networks for Large-Scale Image Recognition. In 3rd International Conference on Learning Representations, ICLR 2015, San Diego, CA, USA, May 7-9, 2015, Conference Track Proceedings, Yoshua Bengio and Yann LeCun (Eds.).

[26] Christian Szegedy, Wei Liu, Yangqing Jia, Pierre Sermanet, Scott Reed, Dragomir Anguelov, Dumitru Erhan, Vincent Vanhoucke, and Andrew Rabinovich. 2015. Going deeper with convolutions. In Proceedings of the IEEE conference on computer vision and pattern recognition. 1-9. https://doi.org/10.1109/CVPR.2015.7298594

[27] Stylianos I Venieris and Christos-Savvas Bouganis. 2017. fpgaConvNet: Automated mapping of convolutional neural networks on FPGAs. In Proceedings of the 2017 ACM/SIGDA International Symposium on FieldProgrammable Gate Arrays. 291-292. https://doi.org/10.1145/3020078. 3021791 
[28] Ying Wang, Jie Xu, Yinhe Han, Huawei Li, and Xiaowei Li. 2016. DeepBurning: Automatic generation of FPGA-based learning accelerators for the neural network family. In 2016 53nd ACM/EDAC/IEEE Design Automation Conference (DAC). IEEE, 1-6. https://doi.org/10.1145/ 2897937.2898002

[29] Qingcheng Xiao, Yun Liang, Liqiang Lu, Shengen Yan, and Yu-Wing Tai. 2017. Exploring heterogeneous algorithms for accelerating deep convolutional neural networks on FPGAs. In Proceedings of the 54th Annual Design Automation Conference 2017. 1-6. https://doi.org/10. 1145/3061639.3062244

[30] Yu Xing, Shuang Liang, Lingzhi Sui, Zhen Zhang, Jiantao Qiu, Xijie Jia, Xin Liu, Yushun Wang, Yi Shan, and Yu Wang. 2019. DNNVM: End-to-End Compiler Leveraging Operation Fusion on FPGA-based CNN Accelerators. In Proceedings of the 2019 ACM/SIGDA International Symposium on Field-Programmable Gate Arrays. 187-188. https://doi. org/10.1145/3289602.3293972

[31] Xuan Yang, Mingyu Gao, Qiaoyi Liu, Jeff Setter, Jing Pu, Ankita Nayak, Steven Bell, Kaidi Cao, Heonjae Ha, Priyanka Raina, et al. 2020. Interstellar: Using Halide's Scheduling Language to Analyze DNN Accelerators. In Proceedings of the Twenty-Fifth International Conference on Architectural Support for Programming Languages and Operating Systems. 369-383. https://doi.org/10.1145/3373376.3378514

[32] Shulin Zeng, Guohao Dai, Hanbo Sun, Kai Zhong, Guangjun Ge, Kaiyuan Guo, Yu Wang, and Huazhong Yang. 2020. Enabling efficient and flexible FPGA virtualization for deep learning in the cloud. In 2020 IEEE 28th Annual International Symposium on FieldProgrammable Custom Computing Machines (FCCM). IEEE, 102-110. https://doi.org/10.1109/FCCM48280.2020.00023

[33] Shijin Zhang, Zidong Du, Lei Zhang, Huiying Lan, Shaoli Liu, Ling Li, Qi Guo, Tianshi Chen, and Yunji Chen. 2016. Cambricon-x: An accelerator for sparse neural networks. In Proceedings of the Annual IEEE/ACM International Symposium on Microarchitecture (MICRO). IEEE, 1-12.
https://doi.org/10.1109/MICRO.2016.7783723

[34] Xiaofan Zhang, Junsong Wang, Chao Zhu, Yonghua Lin, Jinjun Xiong, Wen-mei Hwu, and Deming Chen. 2018. DNNBuilder: an automated tool for building high-performance DNN hardware accelerators for FPGAs. In Proceedings of the IEEE/ACM International Conference on Computer-Aided Design (ICCAD). IEEE, 1-8. https://doi.org/10.1145/ 3240765.3240801

[35] Xiandong Zhao, Ying Wang, Xuyi Cai, Cheng Liu, and Lei Zhang. 2019. Linear symmetric quantization of neural networks for lowprecision integer hardware. In International Conference on Learning Representations.

[36] Xiandong Zhao, Ying Wang, Cheng Liu, Cong Shi, Kaijie Tu, and Lei Zhang. 2020. BitPruner: network pruning for bit-serial accelerators. In 2020 57th ACM/IEEE Design Automation Conference (DAC). IEEE, 1-6. https://doi.org/10.1109/DAC18072.2020.9218534

[37] Shixuan Zheng, Xianjue Zhang, Daoli Ou, Shibin Tang, Leibo Liu, Shaojun Wei, and Shouyi Yin. 2020. Efficient Scheduling of Irregular Network Structures on CNN Accelerators. IEEE Transactions on Computer-Aided Design of Integrated Circuits and Systems 39, 11 (2020), 3408-3419. https://doi.org/10.1109/TCAD.2020.3012215

[38] Li Zhou, Hao Wen, Radu Teodorescu, and David HC Du. 2019. Distributing deep neural networks with containerized partitions at the edge. In 2nd \{USENIX\} Workshop on Hot Topics in Edge Computing (HotEdge 19).

[39] Yanqi Zhou and David Wentzlaff. 2014. The sharing architecture: subcore configurability for IaaS clouds. ACM SIGPLAN Notices 49, 4 (2014), 559-574. https://doi.org/10.1145/2654822.2541950

[40] Barret Zoph, Vijay Vasudevan, Jonathon Shlens, and Quoc V Le. 2018. Learning transferable architectures for scalable image recognition. In Proceedings of the IEEE conference on Computer Vision and Pattern Recognition (CVPR). 8697-8710. https://doi.org/10.1109/CVPR.2018. 00907 